\title{
Parent Perspectives of an Evidence-Based Intervention for Children with Autism Served in Community Mental Health Clinics
}

\author{
Nicole A. Stadnick, \\ San Diego State University/University of California, San Diego Joint Doctoral Program in Clinical \\ Psychology, San Diego, CA, USA. Child and Adolescent Services Research Center, Rady \\ Children's Hospital-San Diego, San Diego, CA, USA
}

Amy Drahota, and Child and Adolescent Services Research Center, Rady Children's Hospital-San Diego, San Diego, CA, USA. Department of Psychiatry, University of California, San Diego, San Diego, CA, USA

\author{
Lauren Brookman-Frazee \\ Child and Adolescent Services Research Center, Rady Children's Hospital-San Diego, San \\ Diego, CA, USA. Department of Psychiatry, University of California, San Diego, San Diego, CA, \\ USA
}

Nicole A. Stadnick: nstadnic@ucsd.edu

\section{Abstract}

Research suggests that improvements to community mental health $(\mathrm{CMH})$ care for children with autism spectrum disorders (ASD) are needed. Recent research examining the feasibility of training $\mathrm{CMH}$ therapists to deliver a package of evidence-based practice intervention strategies (EBPs) targeting challenging behaviors for school-age children with ASD offers initial support for such efforts to improve care. Specifically, quantitative data from a recent pilot study indicate that CMH therapists with limited ASD experience can deliver an EBP intervention with fidelity and perceive it as useful to their practice. Further, client attendance is high and children demonstrate improvement on standardized measures. To further understand the feasibility and impact of training $\mathrm{CMH}$ therapists to deliver EBPs, this mixed-methods study examined parent perspectives of the process and impact of outpatient psychotherapy for 13 parents of children ages 5-13 with ASD whose therapists were trained to deliver the EBP intervention. Results complement and expand previously reported quantitative data on psychotherapy process indicating that parents are highly involved in treatment for their children, perceive a strong therapeutic alliance with their children's therapist, and highlight that treatment was different once therapists began delivering the intervention. Results also indicate themes related to parents' perceptions of positive child and parent outcomes that provide important details on the specific gains that were observed during treatment. Study findings underscore the importance of parent perspectives in understanding the process and impact of implementing EBPs in CMH settings for families of children with ASD.

(C) Springer Science+Business Media, LLC 2012

Correspondence to: Nicole A. Stadnick, nstadnic@ucsd.edu. 


\section{Keywords}

Parent perspectives; Evidence-based practice; Autism spectrum disorders; Community mental health clinics; Challenging behaviors

\section{Introduction}

Autism spectrum disorders (ASDs) are a set of neurobiologically-based disorders characterized by social and communicative difficulties, and exhibition of restricted, stereotyped patterns of behavior or interests (American Psychiatric Association 2000). According to recent estimates, 1 in 110 children have a current diagnosis of ASD in the U.S. (CDC 2009), and an estimated \$35-90 billion is spent annually on care for these children (Ganz 2007). Research on developing methods to deliver effective treatments for children with ASD and their families in community service settings represents a critical public health need given the significant prevalence and societal cost to care for this population.

There are calls for research to enhance dissemination, implementation and sustainability of evidence-based interventions in diverse community settings, and to examine interventions for school-age children with ASD given the lack of research for this age group (Interagency Autism Coordinating Committee 2011). The community mental health (CMH) system (i.e., provides publically-funded mental health services for children with a range of disorders) plays a particularly important role in providing treatment to school-age children with ASD for behavioral and co-occurring psychiatric problems associated with ASD. Most therapists in this service setting report serving a child with ASD, and these children represent, on average, $21 \%$ of their caseloads (Brookman-Frazee et al. 2011c).

While limited, there is growing research on the characteristics of children with ASD in these settings and type of care provided (Brookman-Frazee et al. 2009; Brookman-Frazee et al. 2010). For example, research suggests that this population is typically diagnosed with Asperger's Syndrome or Pervasive Developmental Disorder-Not Otherwise Specified (rather than Autistic Disorder) (Brookman-Frazee et al. 2010; Joshi et al. 2010). These children have a significantly greater number of co-occurring non-ASD disorders relative to youth with non-ASD diagnoses (Joshi et al. 2010). Further, children with ASD are referred for similar problems (challenging behaviors) as children without ASD within the same service system (Brookman-Frazee et al. 2011c; Mandell et al. 2005).

Research examining care provided in CMH settings suggests that gaps exist between EBPs and routine care (Brookman-Frazee et al. 2010). Providers in CMH clinics that are generalist in nature (i.e., not specializing in a particular disorder) do not have sufficient specialized knowledge about ASD to effectively treat this population, and hold a number of inaccurate beliefs about ASD (Heidergerken et al. 2005). In addition, therapists report having limited training in ASD and both parents and therapists experience high levels of frustration due to therapists' limited ASD knowledge to effectively treat this population (Brookman-Frazee et al. 2011a, c).

Research on consumer perspectives, specifically perspectives of parents of children receiving community services, complements and extends data gathered from direct observational methods and provider perspectives of CMH care for children with ASD. Consumer perspectives of care are considered important in efforts to move EBPs to community service settings (Aarons et al. 2009). Understanding consumer perspectives of $\mathrm{CMH}$ care including factors such as client satisfaction and client-therapist alliance is particularly important. Satisfaction is frequently used as an indicator of service quality (Edlund et al. 2003; Lambert et al. 1998), and is considered important in healthcare 
decision-making and evaluation (Kessler and Mroczek 1995). Related, research suggests that parent-therapist alliance predicts parent satisfaction with youth services (Hawley and Weisz 2005). Only a few studies have examined consumer perspectives of community care for children with ASD (Dymond et al. 2007; Kohler 1999; Montes et al. 2009; Renty and Roeyers 2005; Sperry et al. 1999). Even fewer studies exist examining consumer perspectives of specific parent-training interventions (Pillay et al. 2011) or EBPs for children with ASD (Boyd and Corley 2001; Callahan et al. 2008; Dillenburger et al. 2004; Simpson et al. 2007; Valenti et al. 2010). Parent perspectives on treatment process, perceived effectiveness, and specific impacts of treatment are particularly important with this population given that parents and parent advocacy groups play a critical role in service access and policy regarding service provision and funding.

While no known studies have specifically assessed consumer perspectives of EBPs for children with ASD served in CMH settings, Brookman-Frazee et al. (2011a) recently examined the experiences of 23 parents in accessing and receiving routine CMH services for their primarily high functioning, school-aged children and teens with ASD (BrookmanFrazee et al. 2011a). Themes related to parents' experiences receiving care for their children with ASD provided in CMH settings are summarized in Box 1 of Fig. 1. As shown in (A), parents indicated that the primary targets of therapy were challenging behaviors, and $\mathrm{CMH}$ providers' lack of specialized ASD knowledge and "tools" to work with this population greatly impacted parents' perceptions of the process (B) of receiving therapy in this setting. Their perceptions of the therapy process and limited provider ASD knowledge led to (C) dissatisfaction with therapy and perceptions of slow child progress, minimal child gains and high levels of parent and family stress. Findings from Brookman-Frazee et al. (2011a) are consistent with research examining parents of children with ASD who have received a variety of community services (not specifically mental health) in which parents describe dissatisfaction, limited parent-provider collaboration, and the need for improvement in access and receipt of effective care within community service settings (Dymond et al. 2007; Kohler 1999; Montes et al. 2009; Renty and Roeyers 2005; Sperry et al. 1999).

Improving care in this sector is clearly warranted as supported by the research on parents' perspectives of community care. Implementing existing EBPs for ASD (Lord and Bishop 2010; National Standards Report 2009) in CMH clinics offers an innovative solution to the existing "quality gap." Several EBPs for children with ASD have been developed and tested in efficacy studies. There is strong empirical support for child skills training and parentmediated interventions based on principles of applied behavior analysis and cognitive behavioral therapy methods. Unfortunately, existing treatment protocols were not designed for delivery within CMH clinics (i.e., providers with limited ASD experience, traditional $1 \mathrm{~h}$ per week therapy model). EBPs for challenging behaviors in children with ASD are typically "focal intervention practices" (Lord and Bishop 2010) that are not packaged as comprehensive protocols that may be readily adopted for use in mental health clinics.

To address the lack of readily "implementable" EBPs to treat challenging behaviors in children with ASD An Individualized Mental Health Intervention for Children with ASD (AIM HI) (Brookman-Frazee and Drahota 2010) was developed. AIM HI is a package of parent-mediated and child-focused EBP strategies, based on the principles of applied behavior analysis, designed to reduce challenging behaviors in children with ASD ages 5-13 served in CMH clinics. The AIM HI clinical intervention protocol and corresponding therapist training model were developed through a research-community partnership approach in which community stakeholders (organizational leaders, clinicians, and consumers) partnered with ASD intervention experts to integrate findings from a comprehensive mixed-methods needs assessment (Brookman-Frazee et al. 2011a, b, c) and existing EBP strategies for this population (National Standards Report 2009) (see Fig. 1, 
Box 2). The individual components of AIM HI (e.g., functional behavior assessment, use of antecedent and consequence-based strategies, skill modeling, direct skill practice with feedback, environmental modifications for ASD) are classified as "well established" by the National Autism Center's National Standards Project (National Standards Report 2009) and the National Professional Development Center on ASD (Odom et al. 2010), which used rigorous scientific merit rating systems. More specifically, the AIM HI clinical intervention consists of a series of 10 steps (eight required, two as clinically indicated) that are implemented across three treatment phases: Treatment Planning, Active Treatment, and Evaluating Progress. A minimum of 13 sessions is required to complete the protocol although the steps can be repeated if clinically indicated based on child needs and treatment goals. To examine the feasibility of implementing AIM HI in CMH clinics, a recent pilot study was conducted (Brookman-Frazee et al. 2011b) with 13 therapist-child/parent dyads. Therapists were community child mental health therapists who did not specialize in treating children with ASD or using EBP strategies. Participating therapists received an initial allday training in the AIM HI model, and bi-monthly consultation meetings (10 in total) with the AIM HI developers concurrent with their practice with a study family over the 5 months of study participation. Participants included children ages 5-13 years with existing ASD diagnoses and who had been receiving treatment from their participating CMH therapist for less than 1 year. Results of quantitative data indicated that $\mathrm{CMH}$ therapists participated in ongoing ASD training, were able to follow and deliver the AIM HI intervention with a high degree of fidelity, and perceived the intervention as useful. Meaningful reductions in child problem behaviors occurred over 5 months, providing promising support for the AIM HI intervention (see Fig. 1, Box 3).

Quantitative and qualitative data on parent perspectives of their experiences when their child's therapist delivered AIM HI were collected to complement and expand the primary pilot study outcomes (Brookman-Frazee et al. 2011b) and are the focus of the current study. Specifically, the current study examined the perspectives of parents regarding the therapy process and outcomes when therapists delivered AIM HI in CMH clinics.

\section{Methods}

\section{Design}

Data for the current study were collected as part of a pilot study examining the feasibility of training CMH therapists to deliver AIM HI (Brookman-Frazee et al. 2011b). A mixed qualitative and quantitative approach was used to examine parent perspectives of care when therapists delivered AIM HI. Qualitative methods were used due to the desire to best capture the richness and potential diversity of parents' perceptions of their experiences with their child's therapy. These methods are particularly appropriate for gathering participants' indepth, subjective experiences (Marshall and Rossman 2006). Quantitative methods were used to measure parents' perceptions of therapy effectiveness and therapeutic alliance with the child's therapist.

\section{Participants}

Participants included 13 parents of children diagnosed with ASD (85\% of children had Asperger's Disorder or Pervasive Developmental Disorder, Not Otherwise Specified; $15 \%$ had Autistic Disorder, per administrative record) who participated in a pilot study of AIM HI (Brookman-Frazee et al. 2011b). Children qualified for CMH services if they met medical necessity such that their symptoms significantly impacted their functioning and they met criteria for a qualifying diagnosis. Consistent with previous research in these settings (Brookman-Frazee et al. 2011c), children with ASD represented, on average, $24 \%$ of therapists' current caseloads ( $\mathrm{SD}=0.12$; range $8-50 \%$ ). Parents were an average of 43.42 
years $(\mathrm{SD}=6.30$; range $31-53), 54 \%$ had a college degree, and $62 \%$ were married or living with their partner. A total of 11 mothers and two fathers comprised the parent sample. Children were all male, ranged in age from 5 to 13 years $(M=10.27$; $\mathrm{SD}=2.25)$, and were $77 \%$ Caucasian, $15 \%$ Hispanic/Latino, and $8 \%$ Mixed, which is generally consistent with the literature on the population of children with ASD in mental health settings (BrookmanFrazee et al. 2010; Joshi et al. 2010; Mandell et al. 2005). A majority of children (69\%) had co-occurring non-ASD psychiatric diagnoses (per therapist report) comprising: ADHD (39 $\%$ ), Anxiety Disorders (23\%), Disruptive Behavior Disorder (15\%), and Mood Disorders $(8 \%)$. Families attended an average of 12.42 sessions $(\mathrm{SD}=4.56$; range $5-18)$ over the 5 months of study participation. Parent participation was high with parents attending $93 \%$ $(\mathrm{SD}=0.10 ; 73-100 \%)$ of sessions and observational data indicating high levels of withinsession parent involvement (Brookman-Frazee et al. 2011b).

\section{Procedure}

Prior to beginning the pilot study, parents provided written informed consent to participate in study interviews and data collection. Data for the current study included semi-structured interviews and surveys that were administered after families had received 5 months of AIM $\mathrm{HI}$, commensurate with the end of data collection for the pilot study. Interviews were conducted by phone and lasted an average of 10 minutes. Parents also completed two standardized measures at 5-months examining perceptions of the parent-therapist alliance and effectiveness of therapy. Parents received a small monetary compensation for their participation in the larger pilot study that included these interviews. The appropriate Institutional Review Boards approved study procedures.

\section{Measures}

Parent Interview-A trained member of the research team conducted the semi-structured interview. Questions were divided into four primary sections and were semi-structured except for the final question. The first section included a set of questions that asked about parents' experiences in therapy over the past 5 months. Questions included: (1) What was your participation in your child's therapy? (In session? Between-session?) (2) What did you learn about dealing with your child's behavior from your child's therapist? (3) What strategies were most effective in helping you learn? The second section included a set of questions inquiring about parents' perceptions of the impact of therapy on the parent and their child over the past 5 months. These questions included: (1) Were there any changes in your child's behavior? (2) What new skills is your child learning? (3) Have there been any changes in how you deal with your child's behavior? (4) Have there been any changes in your relationship with your child? (5) Have there been any other changes that I have not asked you about? The third section included one question that asked if the parent wanted to share anything further about their experiences in therapy over the past 5 months. The final section included one question that asked about parents' overall satisfaction with therapy over the past 5 months rated on a four-point likert scale ranging from (1) "very" satisfied to (4) "not at all" satisfied. Data were only available for 12 of the 13 participants for this final question due to one parent needing to end the interview early.

Therapeutic Alliance Scale for Children (TASC; Shirk and Saiz 1992)—The TASC (parent version) is a 12-item parent-report measure that examines perceptions of parent-therapist alliance. It was administered to parents after 5 months. Each item is a statement (e.g., "I like spending time with my child's therapist. This statement is...") that parents rate on a four-point likert scale ranging from (1) not like you to (4) very much like you. A Total TASC score is computed based on a composite of all items. Total scores can range from 12 to 48 with higher scores indicating greater perceived therapeutic alliance. The 
TASC has demonstrated strong internal consistency and test-retest reliability in previous studies conducted in CMH settings (Hawley and Weisz 2005).

\section{Perceived Effectiveness Subscale of the Multidimensional Adolescent Satisfaction Scale (Caregiver Report Version) (MASS; Garland et al. 2000a, b)}

-The MASS is a self-report measure that assesses satisfaction with psychotherapy on four dimensions. In the current study, the four-item MASS Effectiveness subscale was used to examine parents' views on the effectiveness of therapy using AIM HI over the past 5 months. Each item is rated on a four-point likert scale ranging from (1) no, not at all to (4), yes, a lot. The mean of the four items produces the score for the Effectiveness subscale that can range from 1 to 4 . Higher scores indicate greater perceived effectiveness. The Effectiveness subscale has demonstrated strong internal consistency in a sample from the same CMH setting (Cronbach's alpha $=.90)$.

\section{Data Analysis}

Analyses of the parent surveys and interview data were first conducted independently. Descriptive statistics were used to analyze data from the MASS, TASC, and the restricted response item regarding overall satisfaction administered during the interview.

Qualitative data were analyzed using a coding, consensus, and comparison methodology (Willms et al. 1990), which followed an iterative approach rooted in grounded theory (Glaser and Strauss 1967). All interviews were first transcribed. Following, several interviews were randomly chosen and examined collectively by two members of the research team to consolidate interview data into analyzable units (i.e., codes). Segments of texts ranging from sentences to paragraphs were assigned codes based on emergent themes (i.e., themes surfacing from the interview text). The final list of codes was established through consensus by all research members and consisted of themes and attitudes related to parents' description of the process of therapy, overall impressions of their experiences in therapy with their child, and the impact of therapy on parent and child outcomes. Codes were divided into two levels of analysis: 1) general (codes at the broadest level of themes), and 2) subthemes (codes at the second level of themes and subsumed within a general theme). Two members of the research team each coded all interviews independently after the final coding list was established. After each interview was independently coded, the two members collectively reviewed each interview to identify and resolve coding discrepancies through discussion. The principal investigator of the study also facilitated resolution of coding discrepancies. This process ensured that each interview had consensus between the coders. Finally, the principal investigator of the study reviewed a randomly selected set of interviews $(n=4)$ to confirm the identified codes agreed upon by the two primary coders. After consensus among the research team was achieved, interview transcripts were then entered, coded, and analyzed in QSR-NVivo 2.0, a program frequently used in qualitative research (Tappe 2002).

Qualitative and quantitative data were integrated through triangulation in order to examine convergence, expansion, and complementarity of the two data sources as suggested by Creswell and Plano Clark (2006). To further contextualize parents' responses and understand the impact of AIM HI, themes identified in the current study were compared to themes related to experiences with $\mathrm{CMH}$ services from the initial parent interviews conducted prior to implementation of AIM HI (Brookman-Frazee et al. 2011a). Participants in the initial study were 23 parents of children (6-19 years) with ASD (high functioning or non-specific ASD diagnoses) who had received or were currently receiving outpatient community-based mental health services. Interviews were semi-structured, approximately $90 \mathrm{~min}$, and consisted of questions examining the following categories: child clinical 
characteristics and clinical histories, service access and experiences with mental health services. These data were analyzed using a similar coding, consensus, and comparison methodology (Willms et al. 1990) described above for the current sample (see BrookmanFrazee et al. 2011a for more details). While the sample size was slightly larger and the interviews were longer due to the broader scope of the initial study, children had similar age and clinical characteristics (e.g., high functioning), families were recruited from the same county and a similar methodology was used to analyze qualitative data. Given these methodological similarities, themes from this prior parent needs assessment are considered a representation of routine care delivered in CMH settings for families of children with ASD and were used as a basis for comparison in the current study. Comparisons of themes are synthesized in Fig. 1 and integrated in the "Discussion". The final themes are described as follows.

\section{Results}

The following are the primary themes that emerged from the data. Descriptions of themes are accompanied by quotes from interview transcripts and survey data to illustrate important themes. Themes are summarized in Box 4 of Fig. 1.

\section{Therapy Process}

High Parent Involvement-All parents reported a high level of active participation within their children's therapy. Specifically, parents explained that they were involved in within-session therapeutic activities. Although the specific types of activities varied across families and over time, parents described their involvement as including reviewing therapy homework, discussing goals, teaching their child skills, and participating in therapeutic activities, all of which are components of the AIM HI clinical intervention protocol. As one parent observed, “....soon after we got into the study...I was actively in the counseling room near the whole time. We were given homework assignments, I'd fill in, worksheets in different things in... [child] and I would be assigned tasks or kind of what we were to work on during that week and then report back, and also to make sure that [child] was keeping up with what he was supposed to be doing. So I would say it was a very active role in his [child's] therapy." In addition, a majority of the parents reported that they were involved in between-session activities, including aiding their children in completing therapeutic homework assignments and tracking their children's challenging behaviors.

\section{Role Play was the Primary Teaching Strategy to Address Challenging}

Behaviors-Regarding the specific content of therapy, a majority of parents reported that the primary teaching strategy used by therapists to teach and practice child skills was roleplay exercises, which often involved the parent. As one parent described, "we practiced previous skills learned and if we would practice a new skill we would take turns, like roleplaying...certain scenarios and the three of us would practice those scenarios.”

\section{Impressions of Therapy}

High Parent Satisfaction and Therapist Considered Effective-Overall, parents described a high level of satisfaction with the therapeutic process and working with the child's therapist. Specifically, parents indicated that therapy was a positive experience and perceived their children's therapists as effective in providing treatment to their children. As one parent explained, “...this is [child's] fourth therapist, and of all the therapists that we have had, I feel like she [current therapist] has been the most beneficial of anyone that we have had. And I'm not...I know she understands autism and ... has helped us more than anyone because she seems to understand him more ... everyone has good suggestions but I feel like she gets [child]." These qualitative data are further supported by the quantitative 
data of general parent satisfaction with AIM HI. Specifically, the mean rating on the parent satisfaction item at the end of the interview was $1.17(\mathrm{SD}=.39$ ), with $100 \%$ of parents reporting that they were satisfied ( $83 \%$ "very"; $17 \%$ "somewhat").

Strong Parent-Therapist Alliance-The quantitative data from the TASC on parent perceptions of their alliance with the therapist confirm and expand the qualitative data. Average parent ratings of parent-therapist alliance were in the high range with the mean score of $40(S D=6.68$; range $28-48)$ out of a possible score of 48 .

\section{Impact of Therapy}

Improved Child Behaviors, Increased Affect Management, and Social SkillsOverall, parents reported positive impacts on the children and on themselves. Specifically, the majority of parents reported that their children's challenging behaviors decreased or positive behaviors increased over the previous 5 months. The two primary skills that a majority of parents reported their children had learned were affect management and social skills. Specifically, parents explained that their children learned how to better regulate their emotions (particularly negative emotions) and implement positive coping strategies. One parent explained: "He [child] has definitely been able to verbalize his emotions more. Verbalize his thought processes and utilize his coping skills. She [current therapist]... worked a lot with him on very, very specific coping skills and specific instances where he was having trouble. And he's...it was really effective, he really responded to her." In addition, parents reported that their children learned conversation and related social skills: "He [child] actually showed a desire, which surprised me greatly, to reach out and become friends with...more fellow students. People he's... actually interested in not just having one friend but having several." Overall, most parents attributed child gains to the therapist and therapy using AIM HI strategies, although a few parents who observed gains were unsure whether to attribute these gains to the therapy or external factors (e.g., other services; typical child development).

Parent survey data were consistent with interview data. Specifically, $93 \%$ of parents reported high mean scores on the MASS Perceived Effectiveness scale, indicating that they perceived therapy as effective in helping their child over the previous 5 months $(M=3.17$; $\mathrm{SD}=.66$; range $2-4$ ).

Parents Learned Skills to Address Child Behaviors-Regarding gains in parent skills, the majority of parents described learning skills from their children's CMH therapist. Some parents described learning more about ASD and strategies to manage ASD symptoms: "I just learned more information on what kind of behaviors people who are diagnosed with Asperger's have and different tips on how to help them... I think the informational strategies were good and then also the organization strategies like [current therapist] put up a chart like on what to do in the morning to get ready. It was actually a visual chart...I'm more aware of what his [child's] symptoms are and how to help him a little bit better from things I've learned."

Other parents described learning skills to manage their children's behavior escalation: "I guess I learned I have a much more expanded repertoire... about things that I can do...We used techniques like diverting attention, starting a new activity, things like that. Really trying them to verbally get him to open up and talk about how he feels in the moment, why he feels that way, as opposed to...before he spiraled out of control..." Parents also reported learning about tracking their children's challenging behaviors and understanding the context in which they occurred: “...paying attention mostly on my part to what happened just before a behavior that we like or didn't like, kind of cause and effect. I never really thought about 
it, I just thought the behavior was there and needed to be changed. It never dawned on me that - well, the reason he did it was because of what happened before it. I found that extremely helpful."

\section{Discussion}

The current study used a mixed-methods approach to examine parents' perspectives of the therapy process and clinical outcomes when CMH therapists delivered AIM HI, a package of EBPs to address challenging behaviors in school-aged children with ASD. These data complement and expand on the previously reported quantitative pilot study data on psychotherapy process (Brookman-Frazee et al. 2011b) by examining the unique perspectives of parents, through qualitative and quantitative methods, regarding the psychotherapy process and impact when their child's CMH therapist delivered AIM HI. Findings of this study yield more specific information on AIM HI delivery and impact on parents and children that may inform efforts to implement these types of interventions to families served in community service contexts. Specifically, findings of this study indicated that parents reported a high level of satisfaction with therapy, perceived a high degree of therapeutic alliance, and considered therapy effective when therapists delivered AIM HI, particularly related to improved child affect management and social skills.

As can be seen in Fig. 1, the therapy process (Box 1, A) was similar for families of children with ASD receiving routine CMH services and those receiving AIM HI (Box 4, A) in that challenging behaviors were the primary targets of therapy (Brookman-Frazee et al. 2011a). However, the therapy process (Box 4, A) was strikingly different for families receiving AIM $\mathrm{HI}$ in their description of high parent involvement in both within- and between-session activities and therapists' use of teaching strategies to introduce and practice skills, specifically role-play, often in which parents were involved.

For parents receiving AIM HI, their impressions of the therapy process affected their overall perceptions of the impact of therapy (Box 4, B), which they described as a positive experience. Their reported positive experiences were often related to their perceptions of their children's therapists as effective in understanding their children's unique characteristics, especially those associated with ASD. These qualitative findings were further substantiated by the quantitative data regarding effectiveness of therapy and therapeutic alliance. Quantitative data indicated that parents reported therapy as effective in helping their children and that they experienced strong parent-therapist alliance. Parent impressions of therapy differ from the reports of parents who received routine $\mathrm{CMH}$ services (Box 1, B) as the latter parents perceived a lack of providers' specialized ASD knowledge and treatment "tools" as contributing to limited child progress (Brookman-Frazee et al. 2011a).

Finally, the majority of parents receiving AIM HI reported that therapy positively impacted child and parent skills (Box 4, C). Specifically, parents explained that their children's challenging behaviors improved and their children learned specific skills-primarily affect management and social skills. Parents also reported learning behavioral skills to improve their interactions with their children. Again, these outcomes are dissimilar from those perceived by families receiving routine CHM services (Box 1, C) who observed minimal child progress in therapy, which also contributed to high levels of parent and family stress (Brookman-Frazee et al. 2011a). Findings regarding the positive impact on child skills for families who received AIM HI are also consistent with the clinical outcomes reported in Brookman-Frazee et al. (2011b) in which meaningful reductions in child challenging behaviors were observed based on standardized measures. 
Results of this study extend the current literature on parent perspectives of EBPs in CMH services, particularly for parents and their children with ASD. The available literature indicates that parents of children with ASD are generally dissatisfied with community services (including CMH services) and there is limited parent-provider collaboration (Brookman-Frazee et al. 2011a; Dymond et al. 2007; Kohler 1999; Montes et al. 2009; Renty and Roeyers 2005; Sperry et al. 1999). However, no studies have assessed parent perspectives of EBPs delivered specifically in $C M H$ settings for children with ASD.

Specifically, parents in this study reported not only a high level of satisfaction with AIM HI, but a majority reported that their children's CMH therapist was effective in understanding how to work with their child with ASD and that they reported a strong alliance with their children's therapist. Parents also reported a positive impact on both child behavior and child and parent skill acquisition throughout delivery of AIM HI. While beyond the scope of the study to examine the relation between parent satisfaction and clinical outcomes, these data suggest that the perception of therapists as effective and a strong therapeutic alliance contributed to both the high level of parent satisfaction and child and parent skill gains. In addition, parents described how they can and are willing to be highly involved in their children's therapy, and commented on their specific involvement within and between therapy sessions when AIM HI was delivered. As parents of children with ASD have called for increased specialized ASD training for community providers and given the critical role that the $\mathrm{CMH}$ system plays in providing treatment services for this population, this study represents an initial step in meeting this growing public health need. Further, this study lends initial support from parents for the acceptability and feasibility of implementing parent-mediated and child-focused EBP strategies for children with ASD served in CMH services and provided by CMH therapists. These data are important because they address potential concerns from therapists or administrators that parents may be unwilling to participate in therapy when therapists use highly directive, behavioral, and manualized interventions.

A few limitations should be noted. First, the small sample size given the pilot study design precludes obtaining a potentially wider range of parent perspectives regarding AIM HI. Although the vast majority reported a positive experience and high satisfaction, a few parents described a neutral experience primarily due to concerns regarding the extent of child progress or attributing child progress to other factors. While these parents were the minority, it is important to recognize the variability in perspectives. In addition, while having data on $\mathrm{CMH}$ services prior to AIM HI implementation was useful in drawing some comparisons in perceptions of service delivery and impact, this study had no simultaneous control group to more rigorously examine parent perceptions of AIM HI. Related, the relation between therapeutic alliance and parent satisfaction with AIM HI and parent and child clinical outcomes could not be assessed given the pilot study sample size. However, this study's qualitative data provide useful information on the specific components of AIM HI that parents perceived as beneficial (e.g., therapist effectiveness in working with their child with ASD). Future larger-scale studies of AIM HI will include a more focused examination of the impact of therapeutic alliance and consumer satisfaction and clinical child and family outcomes.

This mixed methods study was conducted as part of a larger pilot study to examine parent perspectives regarding the implementation of AIM HI by providers in CMH clinics. Providing attention to parents' unique perspectives allowed for specific information on the process and changes in child and parent skills after receiving AIM HI. Specifically, parents reported a high level of satisfaction and parent involvement, perceived their child's CMH therapist as effective in working with children with ASD, and noted a beneficial impact on child and parent skills after receiving AIM HI from their child's CMH therapist for 5 months. It was particularly impressive that parents were able to identify specific teaching 
strategies used by their child's CMH therapist (i.e., role play), and comment on specific skills they and their child had learned (e.g., parent management of child behavioral escalation, child emotion regulation and social skills). Results provide preliminary support for the feasibility and acceptability of implementing EBPs for families of children with ASD served in CMH settings by parents who are critical to moving EBPs to community service settings (Aarons et al. 2009). These results also suggest that continued efforts to implement EBPs may be successful in benefitting child and parent skills and thus overall improving care for families of children with an ASD. Next steps are to conduct a large, randomized controlled trial to more rigorously examine the impact of AIM HI.

\section{References}

Aarons GA, Wells RS, Zagursky K, Fettes DL, Palinkas LA. Implementing evidence-based practice in community mental health agencies: A multiple stakeholder analysis. American Journal of Public Health. 2009; 99(11):2087-2095. [PubMed: 19762654]

American Psychiatric Association. Diagnostic and statistical manual of mental disorders. 4. Washington, DC: Author; 2000. text rev

Boyd RD, Corley MJ. Outcome survey of early intensive behavioral intervention for young children with autism in a community setting. Autism. 2001; 5(4):430-441. [PubMed: 11777258]

Brookman-Frazee L, Baker-Ericzen M, Stadnick N, Taylor R. Parent perspectives on community mental health services for children with autism spectrum disorders. Journal of Child and Family Studies. 2011a Advanced online publication. 10.1007/s10826-011-9506-8

Brookman-Frazee L, Baker-Ericzen M, Stahmer A, Mandell D, Haine RA, Hough RL. Involvement of youths with autism spectrum disorders or intellectual disabilities in multiple public service systems. Journal of Mental Health Research in Intellectual Disabilities. 2009; 2(3):201-219. [PubMed: 19809531]

Brookman-Frazee, L.; Drahota, A. An individualized mental health intervention for children with autism spectrum disorders (AIM HI): A model to address challenging behaviors in children with ASD-A therapist manual. San Diego: University of California; 2010.

Brookman-Frazee, L.; Drahota, A.; Stadnick, N. Training community mental health therapists in a package of evidence-base practice strategies for school-age children with autism spectrum disorders: A pilot study. 2011b. Advanced online publication

Brookman-Frazee, L.; Drahota, A.; Stadnick, N.; Palinkas, LA. Therapist perspectives on community mental health services for children with autism spectrum disorder. Administration and Policy in Mental Health and Mental Health Services Research; 2011c. Advanced online publication

Brookman-Frazee LI, Taylor R, Garland AF. Characterizing community-based mental health services for children with autism spectrum disorders and disruptive behavior problems. Journal of Autism and Developmental Disorders. 2010; 40(10):1188-1201. [PubMed: 20204690]

Callahan K, Henson RK, Cowan AK. Social validation of evidence-based practices in autism by parents, teachers, and administrators. Journal of Autism and Developmental Disorders. 2008; 38:378-392.

Center for Disease Control and Prevention. Prevalence of autism spectrum disorders-Autism and developmental disabilities monitoring network, United States, 2006. Morbidity and Mortality Weekly Report. 2009; 58(SS10):1-20. [PubMed: 19145219]

Creswell, J.; Plano Clark, V. Designing and conducting mixed methods research. Thousand Oaks, CA: Sage Publications; 2006.

Dillenburger K, Keenan M, Gallagher S, McElhinney M. Parent education and home-based behaviour analytic intervention: An examination of parents' perceptions of outcome. Journal of Intellectual \& Developmental Disability. 2004; 29(2):119-130.

Dymond SK, Gilson CL, Myran SP. Services for children with autism spectrum disorders. Journal of Disability Policy Studies. 2007; 18:133-147.

Edlund MJ, Young AS, Kung FY, Sherbourne CD, Wells KB. Does satisfaction reflect the technical quality of mental health care? Health Services Research. 2003; 38:631-645. [PubMed: 12785565] 
Ganz ML. The lifetime distribution of the incremental societal costs of autism. Archives of Pediatric and Adolescent Medicine. 2007; 161(4):343-349.

Garland AF, Aarons GA, Saltzman MD, Kruse MI. Correlates of adolescents' satisfaction with mental health services. Mental Health Services Research. 2000a; 2(3):127-139. [PubMed: 11256722]

Garland AF, Saltzman MD, Aarons GA. Adolescent satisfaction with mental health services: Development of a multidimensional scale. Evaluation and Program Planning. 2000b; 23(2):165175.

Glaser, B.; Strauss, A. The discovery of grounded theory. Chicago, IL: Aldine; 1967.

Hawley KM, Weisz JR. Youth versus parent working alliance in usual clinical care: Distinctive associations with retention, satisfaction, and treatment outcome. Journal of Clinical Child and Adolescent Psychology. 2005; 34(1):117-128. [PubMed: 15677286]

Heidergerken AD, Geffken F, Modi A, Frakey L. A survey of autism knowledge in a health care setting. Journal of Autism and Developmental Disorders. 2005; 35(3):323-330. [PubMed: 16119473]

Interagency Autism Coordinating Committee. 2011 IACC strategic plan for autism spectrum disorder research. 2011. Retrieved from the Department of Health and Human Services Interagency Autism Coordinating Committee website, http://iacc.hhs.gov/strategic-lan/2011/index.shtml

Joshi G, Petty C, Wozniak J, Henin A, Fried R, Galdo M, et al. The heavy burden of psychiatric comorbidity in youth with autism spectrum disorders: A large comparative study of a psychiatrically referred population. Journal of Autism and Developmental Disorders. 2010; 40(11):1361-1370. [PubMed: 20309621]

Kessler RC, Mroczek DK. Measuring the effects of medical interventions. Medical Care. 1995; 33:AS109-AS119. [PubMed: 7723439]

Kohler F. Examining the services received by young children with autism and their families: A survey of parent response. Focus on Autism and Other Developmental Disabilities. 1999; 14:150-158.

Lambert W, Salzer MS, Bickman L. Clinical outcome, consumer satisfaction, and ad hoc ratings of improvement in children's mental health. Journal of Consulting and Clinical Psychology. 1998; 66:270-279. [PubMed: 9583330]

Lord C, Bishop S. Autism spectrum disorders: Diagnosis, prevalence, and services for children and families. Social Policy Report. 2010; 24(1):3-21.

Mandell DS, Walrath CM, Manteuffel B, Sgro G, Pinto-Martin J. Characteristics of children with autistic spectrum disorders served in comprehensive community-based mental health settings. Journal of Autism and Developmental Disorders. 2005; 35(3):313-321. [PubMed: 16119472]

Marshall, C.; Rossman, GB. Designing qualitative research. 4. Thousand Oaks, CA: Sage Publications; 2006.

Montes G, Halterman JS, Magyar CI. Access to and satisfaction with school and community health services for children with autism: National overview. Pediatrics. 2009; 124:S407-S413. [PubMed: 19948606]

National Standards Report. The national standards project-Addressing the need for evidence-based practice guidelines for autism spectrum disorders. Randolph, MA: National Autism Center; 2009.

Odom SL, Collet-Klingenberg L, Rogers SJ, Hatton DD. Evidence-based practices in interventions for children and youth with autism spectrum disorders. Preventing School Failure. 2010; 54(4):275282.

Pillay M, Alderson-Day B, Wright B, Williams C, Urwin B. Autism spectrum conditions-Enhancing Nurture and Development (ASCEND): An evaluation of intervention support groups for parents. Clinical Child Psychology and Psychiatry. 2011; 16(1):5-20. [PubMed: 20223793]

Renty J, Roeyers H. Satisfaction with formal support and education for children with autism spectrum disorder: The voices of the parents. Child: Care, Health and Development. 2005; 32(3):371-385.

Shirk SR, Saiz CC. Clinical, empirical, and developmental perspectives on the therapeutic relationship in child psychotherapy. Development and Psychopathology. 1992; 4:713-728.

Simpson RL, McKee M, Teeter D, Beytien A. Evidence-based methods for children and youth with autism spectrum disorders: Stakeholder issues and perspectives. Exceptionality. 2007; 15(4):203217. 
Sperry LA, Whaley KT, Shaw E, Brame K. Services for young children with autism spectrum disorders: Voices of parents and providers. Infants and Young Children. 1999; 11(4):17-33.

Tappe, A. Using NVivo in qualitative research. Melbourne: QSR International; 2002.

Valenti M, Cerbo R, Masedu F, De Caris M, Sorge G. Intensive intervention for children and adolescents with autism in a community setting in Italy: A single-group longitudinal study. Child and Adolescent Psychiatry and Mental Health. 2010; 4:23-31. [PubMed: 20809976]

Willms DG, Best AJ, Taylor DW, Gilbert JR, Wilson DMC, Lindsay EA, et al. A systematic approach for using qualitative methods in primary prevention research. Medical Anthropology Quarterly. 1990; 4:391-409. 


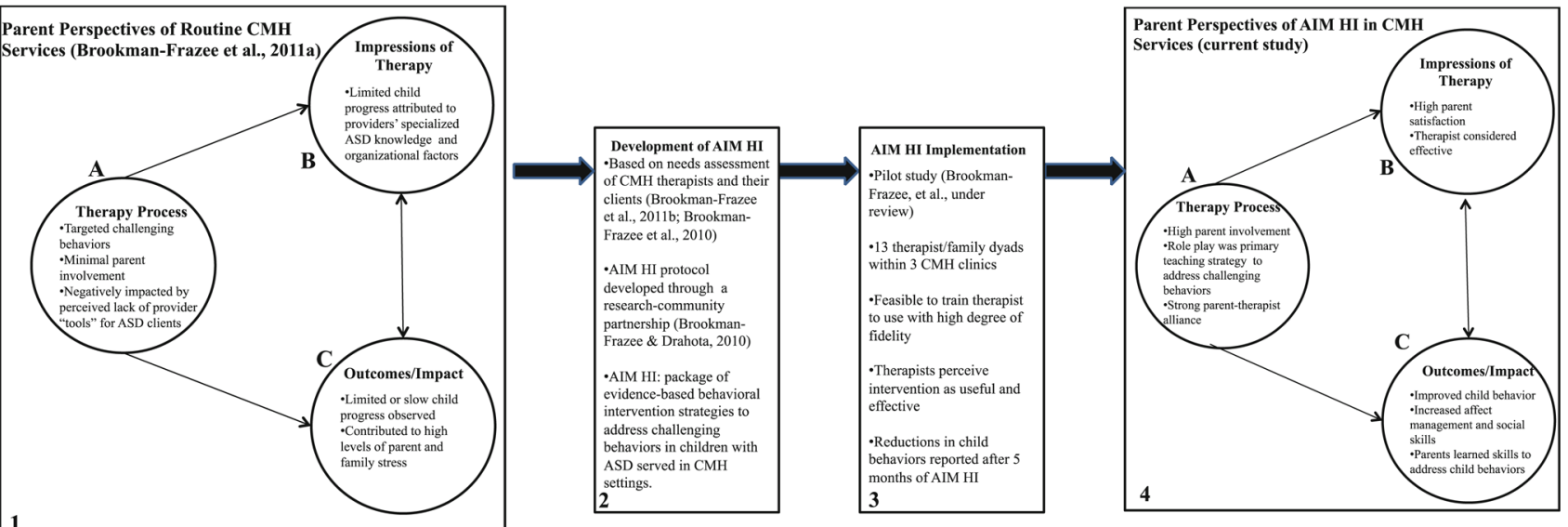

Fig. 1.

Parents' perspectives of community mental health services prior to and following implementation of AIM HI 